\title{
Methods for reducing delays in the diagnosis of multiple myeloma
}

\author{
Constantinos Koshiaris*,1 \\ ${ }^{1}$ Nuffield Department of Primary Care Health Sciences, University of Oxford, Oxford, UK \\ *Author for correspondence: constantinos.koshiaris@phc.ox.ac.uk \\ "Prediction rules can be used to estimate the risk of a patient based on their clinical presentation \\ and have the potential to be automated within software systems in order to flag-up high-risk \\ patients"
}

First draft submitted: 30 December 2018; Accepted for publication: 17 January 2019; Published online: 26 February 2019

Keywords: blood tests $\bullet$ clinical prediction rules $\bullet$ diagnostic delay $\bullet$ hematology $\bullet$ multiple myeloma $\bullet$ myeloma $\bullet$ primary care

\section{Multiple myeloma \& diagnostic delays}

Myeloma is a hematological malignancy that starts in the bone marrow and mainly affects the elderly population with a median diagnosis age of 70 years old [1]. A systematic review has shown that patients with multiple myeloma experience long diagnostic intervals, with $50 \%$ of patients having waiting times longer than 3 months [2]. Myeloma patients also experience many consultations as 50\% visit their primary care physician more than three times before they get referred to specialist services [3]. Longer time to diagnosis has been associated with more complications and worse disease-free survival [4]. Approximately 30\% of myeloma patients are being diagnosed through emergency services and these patients have worse survival compared with patients being diagnosed through other routes [5,6]. More timely diagnosis could have a positive impact on myeloma patients both in terms of treatment options and subsequent survival and quality of life.

Myeloma has been classified as one of the hardest cancers to diagnose and no single reason is responsible for the delays observed in diagnosis [7]. It is usually a combination of multiple factors which together form a very complex diagnostic picture. One of them is the rarity of the disease; in the UK, the yearly incidence is approximately nine per 100,000 population and it accounts for $2 \%$ of all cancer cases diagnosed every year [8]. A full-time primary care practitioner in the UK will diagnose on average one myeloma case every 5 years. The other main factor is the nonspecific nature of the symptoms that can include back pain, bone pain, fatigue and repeated infections [9]. These symptoms are also common in many other conditions of varying severity that makes it difficult to suspect myeloma as the underlying cause. Patients with comorbidities are also more likely to experience diagnostic delays as multiple comorbidities can mask myeloma-related symptoms [10].

\section{Methods for reducing delays in myeloma}

Currently, no screening programs exist for myeloma as it can lead to overtesting and overdiagnosis of monoclonal gammopathies of undetermined significance (MGUS). MGUS is a condition that precedes myeloma, and is not actually treated but monitored [11]. The risk of progression from MGUS to symptomatic myeloma is approximately $1 \%$ per year and the prevalence of MGUS in adults over 50 years old is estimated to be approximately 3\% [11,12]. Over-diagnosing MGUS can have a big burden on the healthcare system; MGUS patients require annual life-long monitoring, but the risk of progression to symptomatic myeloma that requires treatment is very small. A US study estimated that the annual cost for MGUS monitoring is at least $\$ 109$ million [13]. The presence of MGUS can also have a significant impact on the quality of life [14]. Given the above reasons, it is likely that a screening program might cause more harm to both patients and the healthcare system. Thus, myeloma screening is not a viable method, necessitating the development of alternative detection strategies. 
The biggest opportunity to detect myeloma is to investigate patients with symptoms once they present in healthcare services. Primary care plays one of the most important roles for the early diagnosis of myeloma as it is usually the first point of contact between the patient and the healthcare system. General practitioners (family doctors) are responsible for decisions regarding referral to secondary care. Prompt suspicion of the disease can result in more timely referrals to the appropriate departments and thus reduce time to diagnosis. Interventions implemented at the primary care level can be quite effective for early diagnosis of myeloma.

In most cancers, symptoms can be quite indicative of the diseases, for example, rectal bleeding and colorectal cancer or hematuria and bladder cancer. These types of symptoms, which are also referred to as 'red flags' in most cases, result in urgent investigation and referral to specialist services. Unfortunately, myeloma symptoms on their own are not very useful due to how common they are. Most myeloma symptoms are not predictive, with most of them having a positive predictive value of less than $1 \%$ [15]. They become more predictive when combined with abnormalities detected in blood tests such as the full blood count, creatinine, calcium and inflammatory markers [15]. Blood test combinations can be quite indicative of myeloma and different testing strategies can be used in order to rule in or rule out myeloma. A recent study has shown that certain test combinations like the erythrocyte sedimentation rate and hemoglobin can be used to rule out myeloma if found normal in patients presenting with myeloma-related symptoms, such as bone pain; abnormalities in these tests should result in more specialist investigations [16]. Given the importance of blood tests in myeloma, it is important that clinicians should have easy access to common blood tests.

Another alternative approach that could be used is the development of tools that can help clinicians flag high-risk patients for myeloma. These tools usually take the form of clinical prediction rules. In the last decade, there has been a big increase in the development of prediction rules that can be used to diagnose cancer [17]. This was made possible only recently due to the rise of electronic health record databases, which allow for the development of prediction rules in adequately powered samples, as well as the increase in computational power, which allows the analysis of such big databases [18]. Prediction rules can be used to estimate the risk of a patient based on their clinical presentation and have the potential to be automated within software systems in order to flag-up high-risk patients. Electronic trigger-based interventions have been shown to significantly reduce time to diagnosis in other cancers such as colorectal and prostate [19]. Given that, in many countries, patient information is integrated within information and software systems; this approach has the potential to become a very useful and efficient tool for the early diagnosis of cancer.

\section{Conclusion \& recommendations for future research}

Some progress has been made in recent years on early diagnosis of myeloma, but there is still plenty of room for improvement. Based on current evidence, the best way to currently identify high-risk patients is through the appropriate use of common blood tests like the full blood count, inflammatory markers and calcium. Given that the incidence rate has been increasing, it is very important that more research is done on early diagnosis of myeloma [20]. Prediction rules using electronic health records data from primary care could prove to be a strong additional support tool for clinicians to identify myeloma. Future research should focus on their development, validation and implementation.

Financial \& competing interests disclosure

C Koshiaris is funded by the Primary Care Research Trust, the University of Oxford, and National Institute for Health Research (NIHR) Oxford CLAHRC. The views expressed are those of the author and not necessarily those of the NHS, the NIHR, or the Department of Health. The author has no other relevant affiliations or financial involvement with any organization or entity with a financial interest in or financial conflict with the subject matter or materials discussed in the manuscript apart from those disclosed.

No writing assistance was utilized in the production of this manuscript.

Open access

This work is licensed under the Attribution-NonCommercial-NoDerivatives 4.0 Unported License. To view a copy of this license, visit http://creativecommons.org/licenses/by-nc-nd/4.0/

\section{References}

1. Zweegman S, Palumbo A, Bringhen S, Sonneveld P. Age and aging in blood disorders: multiple myeloma. Haematologica 99(7), 1133-1137 (2014). 
2. Koshiaris C, Oke J, Abel L, Nicholson BD, Ramasamy K, Van den Bruel A. Quantifying intervals to diagnosis in myeloma: a systematic review and meta-analysis. BMJ Open 8(6), e019758 (2018).

3. Lyratzopoulos G, Neal RD, Barbiere JM, Rubin GP, Abel GA. Variation in number of general practitioner consultations before hospital referral for cancer: findings from the 2010 National Cancer Patient Experience Survey in England. Lancet Oncol. 13(4), 353-365 (2012).

4. Kariyawasan CC, Hughes DA, Jayatillake MM, Mehta AB. Multiple myeloma: causes and consequences of delay in diagnosis. QJM 100(10), 635-640 (2007).

5. Elliss-Brookes L, McPhail S, Ives A et al. Routes to diagnosis for cancer - determining the patient journey using multiple routine data sets. Br. J. Cancer 107(8), 1220-1226 (2012).

6. Howell D, Smith A, Appleton S et al. Multiple myeloma: routes to diagnosis, clinical characteristics and survival - findings from a UK population-based study. Br. J. Haematol. 177(1), 67-61 (2017).

7. Lyratzopoulos G, Wardle J, Rubin G. Rethinking diagnostic delay in cancer: how difficult is the diagnosis? BMJ 349, g7400 (2014).

8. Cancer Research UK. Myeloma incidence statistics. (2015). www.cancerresearchuk.org/health-professional/cancer-statistics/statistics-by-cancer-type/myeloma/incidence

9. Hsu DC, Wilkenfeld P, Joshua DE. Multiple myeloma. BMJ344, d7953 (2012)

10. Friese CR, Abel GA, Magazu LS, Neville BA, Richardson LC, Earle CC. Diagnostic delay and complications for older adults with multiple myeloma. Leuk. Lymphoma 50(3), 392-400 (2009).

11. Kyle RA, Rajkumar SV. Management of monoclonal gammopathy of undetermined significance (MGUS) and smoldering multiple myeloma (SMM). Oncology (Williston Park) 25(7), 578-586 (2011).

12. Wadhera RK, Rajkumar SV. Prevalence of monoclonal gammopathy of undetermined significance: a systematic review. Mayo Clin. Proc. 85(10), 933-942 (2010).

13. Go RS, Swanson KM, Sangaralingham LR, Habermann EB, Shah ND. Clinical prevalence (diagnosed cases) of monoclonal gammopathy of undetermined significance in the US: estimating the burden on health care. Leukemia 30(6), 1443-1446 (2016).

14. Hildebrandt MAT, Callender RA, Belachew AA et al. Quality of life and cancer worry in a follow-up cohort of patients with asymptomatic monoclonal gammopathies. J. Clin. Oncol. 36(15_suppl), 8049 (2018).

15. Shephard EA, Neal RD, Rose P, Walter FM, Litt EJ, Hamilton WT. Quantifying the risk of multiple myeloma from symptoms reported in primary care patients: a large case-control study using electronic records. Br. J. Gen. Pr. 65(631), e106-e113 (2015).

16. Koshiaris C, Van den Bruel A, Oke JL, Nicholson BD, Shephard E, Braddick M et al. Early detection of multiple myeloma in primary care using blood tests: a case-control study in primary care. Br. J. Gen. Pract. 68(674), e586-e593 (2018).

17. Hippisley-Cox J, Coupland C. Symptoms and risk factors to identify men with suspected cancer in primary care: derivation and validation of an algorithm. Br. J. Gen. Pract. 63(606), 1-10 (2013).

18. Herrett E, Gallagher AM, Bhaskaran K et al. Data resource profile: Clinical Practice Research Datalink (CPRD). Int. J. Epidemiol. 44(3), $827-836$ (2015).

19. Murphy DR, Wu L, Thomas EJ, Forjuoh SN, Meyer AN, Singh H. Electronic trigger-based intervention to reduce delays in diagnostic evaluation for cancer: a cluster randomized controlled trial. J. Clin. Oncol. 33(31), 3560-3567 (2015).

20. Vélez R, Turesson I, Landgren O, Kristinsson SY, Cuzick J. Incidence of multiple myeloma in Great Britain, Sweden, and Malmö, Sweden: the impact of differences in case ascertainment on observed incidence trends. BMJ Open 6, e009584 (2016). 
